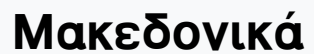

Tóp. 11, Ap. 1 (1971)

MAKGДONIKA

¿YГГPAMMA IIEPIOAIKON
THE ETAIPEIAZ MAKEAONIKSN EMOYAN

EmIMEAEIA,

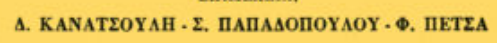

TOMOE EN $\triangle$ EKATOE

1971

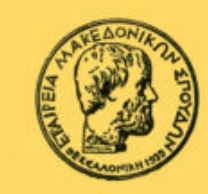

EN EEIIAAONIKH

1971

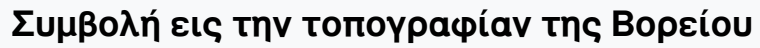


aıćvas

\section{Vëra Hrochovâ}

doi: $\underline{10.12681 / \text { makedonika.948 }}$

\section{Copyright $\odot$ 2014, Vëra Hrochovâ}

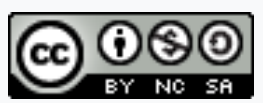

Ađ¿ıı Xpท́бnৎ Creative Commons Attribution-NonCommercial-ShareAlike 4.0.

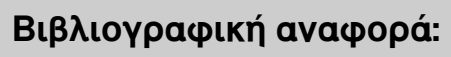

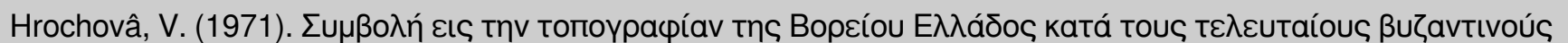
aı́́vac. Макєঠovıкá, 11(1), 376-394. https://doi.org/10.12681/makedonika.948 


\section{BEITRAG ZUR TOPOGRAPHIE NORD-GRIECHENLANDS IN DER SPÄTBYZANTINISCHEN ZEIT}

Die Entwicklung und Bedeutung der byzantinischen Stadt gehört seit dem Münchener Byzantinisten-Kongress 1958 zu den intesiv diskutierten und analysierten Fragen der gegenwärtigen Forschung der Historiker. Wesentliche Meinungsunterschiede haben sich dabei selbst in der Definition der byzantinischen Stadt gezeigt, denn es war schon seit dem 19. Jahrhundert klar, dass keine der so heftig und oft auch dogmatisch diskutierten Definitionen der west - und mitteleuropäischen Stadt für Südosteuropa angewandt werden kann. Es wäre jedoch falsch, den west-europäischen Stadtbegriff als Masstab für alle zu betrachten und daher auf jede genauere allgemeine Bestimmung der südosteuropäischen (und auch osteuropäischen) Stadt verzichten zu wollen. Andererseits sieht die moderne Forschung in der Stadt-Begriff-Diskussion kein Endziel ihrer Bemühung, sondern nur einen Ausgangspunkt, der für die allgemeine Verständigung der Historiker dringend notwendig ist ${ }^{1}$.

Eine nähere Bestimmung des städtischen Phänomens in Byzanz ist bekanntlich durch die äusserst spärlich erhaltene Quellenunterlage sehr erschwert. Daher die Gefahr, dass man aus zerstreuten Einzelangaben, die uns zufällig erhalten geblieben sind, einen "Typus» kombiniert, ohne seine Anwendbarkeit nachprüfen zu können, besonders wenn dieser - wie es vor allem in der älteren Forschung geschah-aufgrund der Angaben für Konstantinopel und eventuell auch für Thessaloniki konstruiert wird; denn diese grossen Emporien unterschieden sich ganz wesentlich von den meisten städtischen; bzw. stadtähnlichen Siedlungen in Byzanz und besonders dann in der spätbyzantinischen Zeit im 13.-15. Jahrhundert. Bessere Resultate kann man nur dann erreichen, wenn man die Gesamtheit der byzantinischen Städte (oder sogenannten «Städte») und ihre Beziehung zur feudalen Gesellschaft in Betracht nimmt. Einen wichtigen Schritt in dieser Richtung hat der sowjetische Historiker Kaschdan für die frühere Entwicklungsperiode der byzantinischen Städte unternommen ${ }^{2}$. Interessante Hinweise bringt neuerdings auch die griechische Forscherin B. Papoulia, wenn sie die Stadt im Rahmen der Stadt-

1. Dies hat M a x W e b e r in seiner berühmten Arbeit: Die Stadt. Begriff und Kategorien, «Archiv für Sozialwissenschaft u. Sozialpolitik», Bd. 47 (1921), S. 621 ff. gezeigt.

2. A. P. K a s ch d a n, Děrevnja i gorod v Vizantii IX-X vv., Moskva 1960. 
Land Beziehung verstehen will. Sie betont dabei drei wesentliche Kriterien ${ }^{1}$ : 1. die soziale Mobilität (sowohl die vertikale, wie auch die horizontale), 2. «die kulturelle Ausstrahlung», 3. die ökonomischen Beziehungen. Wenn unter der «kulturellen Ausstrahlung» auch die politische und kirchliche Verwaltung begriffen sind, könnten wir diesen kriterien zustimmen, auch wenn wir die ökonomischen Beziehungn als primär betrachten. Auch bei einer solchen, eher typologischen Bestimmung können wir nicht ohne weiteres mit der lükenhaften Quellenbasis fertig werden. Besonders für die soziale Mobilität und für die gewerblichen und handwerklichen Bestandteile der ökonomischen Tätigkeit konnen wir in den Quellenangaben nur ausnahmsweise vereinzelte direkte Belege finden. Sollen wir deswegen an jede Erkenntnismöglichkeit verzichten?

Es gibt einen Weg, der bisher nocht nicht gründlich untersucht und ausgenutzt wurde; nämlich den, der eine systematische grossräumige Auswertung der indirekten Hinweise kombiniert mit den eindeutigen Quellenangaben versuchen würde. Dabei möchten wir im voraus auf den scholastischen Streit verzichten, ob es sich in diesem oder jenem Falle «schon» um eine Stadt, oder «noch» um ein Dorf, Marktdorf usw. handelt, sondern nach den gesellschaftlichen, nichtlandwirtschaftlichen Funktionen und ihrer Lokalisierung fragen. In der byzantinischen und insbesondere in der spätbyzantinischen Gesellschaft haben wir es mit einem hochentwickelten Typus der Gesellschaft des Mittelalters zu tun, in welchem Warenproduktion und Geldwirtschaft weit über den Rahmen der einfachen landwirtschaftlichen Produktion und der Naturalwirtschaft greifen und dementsprechende lokalisierte ausseragrarische Funktionen in Anspruch nehmen. Zugleich handelt es sich aber um eine Gesellschaft, in welcher die territoriale Trennung zwischen der agrarischen und nichtagrarischen Produktion (die zweite gesellschaftliche Arbeitsteilung) nicht durchgeführt wurde. Ausserdem hatte die byzantinische Gesellschaft eine ausgebreitete politische und kirchliche Verwaltung — und zwar nicht nur in der Hauptstadt, sondern auch in der Provinz. Die Zentren dieser Verwaltung waren nicht selten zugleich als Festungen bekannt. Wir konnen also zusammenfassend sagen, dass wir die Untersuchung der städtischen Siedlungen als eine Untersuchung der lokalisierten ausseragrarischen, nichtlandwirtschaftlichen Funktionen unternehmen werden.

Wir werden besonders folgende Funktionen in Betracht nehmen: die Vermittlung des Handels, sei es in der Ebene des lokalen Marktes oder des internationalen Fernhandels;

1. B. P a pouli a, Stadt und Land in Byzanz, Actes du Ile Congrès International des Études du Sud-Est Européen (Athènes 7-13 Mai 1970), I. Histoire, Rapport, S. 6. 
die handwerkliche Produktion in den Zweigen, welche den Rahmen und das Ausmass eines einfachen Dorfhandwerks überschreiten;

die Bedienung und Vermittlung des Transports;

die zivile administrative Verwaltung;

die kirchliche Verwaltung;

die militärische Funktion.

Die hier angeführten Funktionen kann man nicht in einem gleichwertigen Nebeneinander und gegenseitig isoliert sehen. Sie bildeten untereinander ein System der Abhängigkeiten und müssten sich oft kombiniert haben. Eine solche Kombination, Koppelung der Funktionen ist mancherorts in den Quellen belegt, manchmal dürfen wir sie jedoch eher vermuten als belegen. So dürfen wir z. B. in einem stabilen Zentrum der politischen Verwaltung meistens einen gewissen Anteil der breiteren Marktbeziehungen voraussetzen, ähnlich wie auch in einer Festung, sofern es sich nicht um eine Burg des frankischen Charakters handelte. Ein Mittelpunkt des Fernhandels, soweit er schon mehr als eine Durchgangsstation bedeutete, ist ohne Beziehungen zum Lokalmarkt kaum denkbar.

Es sollte hier also untersucht werden, wo sich auf einem grösseren Territorium die lebenswichtigen nichtlandwirtschaftlichen Funktionen verwirklicht haben, bzw., wie sich diese Funktionen und ihre Lokalisierung während der Jahrhunderte entwickelt hatten. Dieser Vorgang möchte also nicht bei den einzelnen Ortschaften prüfen, ob diese einem im voraus konstruierten Stadtbegriff entsprechen, sondern fragen, welche ausseragrarische Funktionen in diesen Ortschaften bewiesen sind, bzw. auch rekonstruiert und vorausgesetzt werden konnen. Wenn wir doch den traditions-belasteten Begrif Stadt auch weiter benutzen werden, dann für diejenigen Orte, in denen sich mehrere ausseragrarische Funktionen konzentriert haben, ausnahmsweise auch für diejenigen, welche in den Quellen konsequent als Stadt genannt wurden. Es wird dabei vorausgesetzt, dass auch solche Lokalitäten zum grossen Teil oder vielleicht überwiegend von einer Agrarbevölkerung bewohnt waren, was übrigens keine Besonderheit der byzantiniscen Kleinstädte (z. B. verglichen mit Zentraleuropa) bedeutet. Die Aussagefähigkeit solcher Untersuchungen wird dadurch vorausgesetzt, bzw. mitbestimmt, ob wir imstande sind, ein möglichst grosses und geschlossenes Territorium während eines längeren Zeitabschnittes zu untersuchen. Bei dem gegebenen Stand der Forschung müssen wir uns je doch mit einer Stichprobe für Griechenland begnügen. Wir werden in diesem Aufsatz die «städtische» Entwicklung in einer der byzantinischen Regionen untersuchen - in Thessalien.

Dabei soll ein Versuch unternommen werden, die heute erreichbaren Angaben über ausseragrarische Funktion der einzelnen thessalischen Ort- 
schaften in der spätbyzantinischen Zeit (im 13.-15. Jahrhundert) zusammenzustellen, berücksichtigend dabei eventuelle Veränderungen dieser Funktionen verglichen mit den früheren Jahrhunderten. Eine zentrale Bedeutung werden dabei für uns die Angaben schriftlicher Quellen haben; die materiellen Uberreste nur sofern sie an der Oberfläche erhalten und von den $\mathrm{Au}-$ genzeugen registriert sind; denn die mittelalterliche Archäologie hat auf diesem Gebiet nur sehr geringe Schritte getan.

Thessalien bildete ein geschlossenes Ganzes nicht nur im geographischen, sondern verhältnismässig lange auch im politischen Sinne. Die relative, politische Stabilität ermöglicht es uns, die Entwicklung der städtischen Besiedlung von der inneren Struktur des Gebietes her zu beobachten. Auch wenn Thessalien gegenüber den Nachbarlandschaften - sei es Epirus, Makedonien oder Mittelgriechenland - durch schwer zugängliche Gebirgsketten abgegrenzt war, bildete sie kein geographisch völlig einheitliches Territorium. Ihre einzelnen Teile unterschieden sich voneinander nicht nur durch die Naturbedingungen, sondern auch durch den Charakter der Besiedlung und der Lebenshaltung der Einwohner. Die fruchtbaren Gebiete lagen in der nordöstlichen Ebene unter dem Nieder-Olymp, im Osten an dem Volos-Golf und im Binnenland dann südlich des oberen Flusses von Enipeus und in der westlichen Ecke Thessaliens am Oberlauf von Peneios und Trikkalinos. Die fruchtbaren Gebiete im Westen und im Osten waren durch das sümpfige Binnenland am Mittellauf des Peneios und durch das mittelthessalische Hügelland getrennt. Für eine wirtschaftliche Charekteristik der weniger fruchtbaren und gebirgigen Gebiete im Binnenland und in den Randgebieten Thessaliens sind wir aus dem Mittelalter sehr wenig informiert. Mit Recht wird vorausgesetzt, dass es sich um Gebiete handelte, wo die Hirtenwirtschaft eine wichtige Rolle in der Erwerbstätigkeit der Bevölkerung gespielt hat. Wenden wir jetzt unsere Aufmerksamkeit der städtischen Besiedlung der einzelnen Regionen Thessaliens $\mathrm{zu}^{1}$.

Der nördliche Teil Westthessaliens bildete eine ziemlich geschlossene Landschaft. Ihr zentraler Teil - am oberen Peneios und Trikkalinos - wird von den Zeitgenossen als sehr fruchtbar beschrieben (mit Produktion von Getreide, Wein und Früchten) ${ }^{2}$. Durch dieses Gebiet führte ein alter Handels-

1. A. Philipps o n, Die Griechischen Landschaften, Bd. I, 1: Der Nordosten der Griechischen Halbinsel. Thessalien und die Spercheios - Senke. Nebst einem Anhang: Beiträge zur historischen Landeskunde Thessaliens von Ernst Kirsten, Frankfurt/M 1950.

2. A. Phili p p s o n, I, 1, S. 43 ff; W. T o m a s c h e k, Zur Kunde der Hämus-Halbinsel. Die Handelswege im 12. Jahrhundert nach den Erkundigungen des Arabers Idrisi, II. Sitzungsberichte der Philosoph-histor. Cl. der Kaiserl. Akad. der Wiss., 113, Wien 1886, S. 285-373; weiter nur Idrisi, S. 348 ff. 
weg, die wichtigste und zeitweilen einzige Verbindung zwischen Thessalien und Epirus mit Abzweigungen nach Albanien und Westmakedonien. Das Verwaltungszentrum lag in Trikkala und hat sich dort auch im 14. Jahrhundert gehalten ${ }^{1}$. Im 10. Jahrhundert war hier auch ein Bischofssitz ${ }^{2}$, bei Idrisi wird Trikkala als ein Städtchen in einer fruchtbaren Landschaft beschrieben ${ }^{3}$. Auch nach der Eroberung durch Stephan Duschan 1358 blieb die Funktion des politischen Zentrums der Verwaltung erhalten. Die administrative Funktion hat sich hier also mit jener der Handels - und Transportstation gekoppelt; der Lokalmarkt für die fruchtbare landwirtschaftliche Umgebung darf wolf vorausgesetzt werden, kaum aber eine ausgebreitetere handwerkliche Produktion für den Markt. Dort, wo das Peneiostal schon in das Vorgebirge von Chassia eindrigt, lag das kirchliche Zentrum des spätmittelalterlichen Thessaliens im Westen der Bischofssitz Stagoi. Bis in das 13. Jahrhundert hinein wissen wir in diesem Ort nur von dem Sitz eines Bischofs und der Verwaltung einer feudalen Domäne ${ }^{4}$. Kantakuzenos spricht jedoch von einer $\mathrm{Fe}-$ stung ( $\varphi$ poúpıov) Stagoi im Jahre 1333 und drei Jahre später wird in einer Urkunde ein zum Bischoftum gehörender Markt erwähnt, der entweder direkt im Ordoder in seiner Nähe lag $^{5}$. Wir haben hier also mit einer Ausbreitung der nichtlandwirtschaftlichen Funktionen zu tun bei einem Ort, in dessen Nähe sich der Handelsweg aus Epirus mit jenem aus Westmakedonien traf. Es war ausgerechnet in der Umgebung von Stagoi, wo der neue Klosterkom-

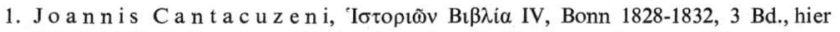

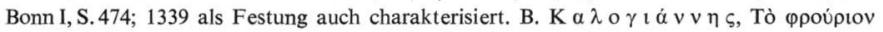

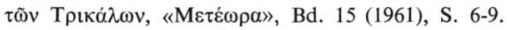

2. H. G e l z e r, Ungedruckte und ungenügend veröffentlichte Texte der Notitiae episcopatuum, ein Beitrag zur byzantinischen Kirchen - und Verwaltungsgeschichte. Abhandlungen der philos-philolog. Cl. der Königl. Bayer. Akad. der Wiss., Bd. 21, München 1901, S. 557.

3. W. T o m a s c h e k, Idrisi, S. 348 ff: neue archäologische Ausgrabungen: E ̉. Ko $\rho-$

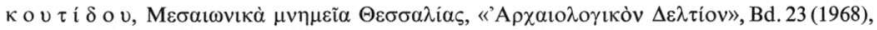

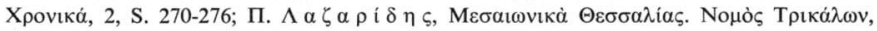

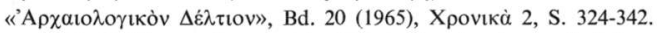

4. F. D ö $1 \mathrm{~g}$ e r, Regesten der Kaiserurkunden des oströmischen Reiches II, S. 23, München 1925; H. G el z e r, Ungedruckte, S. 557; 1328 gehörte den Feudalen Gabrielopouli, A. P h i 1 i p ps o n, I, 1, S. 280; C. As tru c, Un document inédit de1163 sur l'évéché thessalien de Stagi. Parisinus Suppl. gr. 1371, «Bulletin de Correspondance Hellénique», Bd. 83 (1919), S. 206-246.

5. F. D öl g e r, Regesten IV, S. 159, Cant., Bonn., I, S. 474; $\Sigma$. 'A $\rho \imath \sigma \tau \dot{\alpha} \rho \chi \circ v$, X $\rho v-$

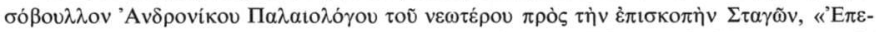


$\lambda \varepsilon 1$, Bd. 3 (1866-67), S. 32-38; Stagoi im 14. Jhr.; I. K. B o $\gamma \iota \alpha \tau \zeta$ i $\delta \eta \varsigma$, Tò Xpovıкòv $\tau \tilde{v} v$

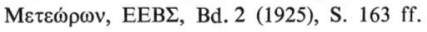


plex Meteora im 14. Jahrhundert entstanden ist ${ }^{1}$. Unklar bleibt, ob die in einer Urkunde vom Jahre 1321 erwähnte und mit mehreren Mühlen ausgestattete Ortschaft Raxa ${ }^{2}$ mit jenem nördlich von Trikkala liegenden Raxa identisch ist, über dem Kirsten eine auf dem Berg liegende alte Stadtsiedlung vermutet ${ }^{3}$.

Südlich vom Peneiosfluss war der westliche Teil Thessaliens schon seit dem Altertum in der Ebene dicht besiedelt. Neben Getreideanbau hat in der Richtung gegen Südosten die Viehzucht zugenommen. Aus der Antike überlebten in dieser Gegend zwei politische und wirtschaftliche Zentren, Gomphoi und Metropolis. Das erste von beiden wird in den Quellen der spätbyzantinischen Zeit nicht mehr erwähnt und auch die Überreste scheinen anzudeuten, dass es untergegangen ist ${ }^{4}$. Auch Metropolis überlebte augenscheinlich die frühbyzantinische Periode nicht als Stadt und wurde dann vergessen $^{5}$. Es hat sich jedoch in der spätbyzantinischen Zeit in der Nähe der antiken Gomphoi ein neues Zentrum entwickelt - Phanarion, das wir aus der früheren Zeit als Festung und Zentrum der kirchlichen Verwaltung kennen ${ }^{6}$. In der stürmischen Zeit am Anfang des 14. Jahrhunderts öfter den Herrn wechselnd, hat Phanarion im Jahre 1334 von dem dort damals herrschenden Geschlecht der Gabrielopouloi ein wichtiges Privilegium gewonnen, das zu den wenigen gehört, welche uns erhalten geblieben sind. Diesem Privilegium nach bekamen die führenden Schichten der Stadt wichtige Verwaltungsund Steuerermässigungen, darunter einige auch Befreiung von Marktgebühren $^{7}$. Es wird in der Urkunde keine Marktproduktion direkt erwähnt; doch darf sie hier mit Sicherheit für die landwirtschaftliche Produktion vorausgesetzt werden. Wenn die von E. Kirsten gezeigte Parallele zwischen Phanarion

1. D. M. N i c o l, Meteora, The Rock Monasteries of Thessaly, S. 2 10; W. W. Hy d e, The Monasteries of Meteora and greek Monasticism, «Bulletin of the Geographical Society

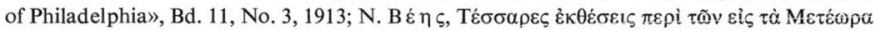

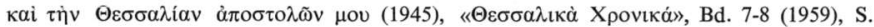

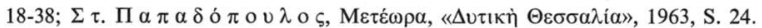

2. F. D ölg e r, Regesten IV, S. 147;

3. A. Phili p p son, I, 1, S. 275.

4. Ebda, S.56; F. St äh 1 i n, Inschriften aus Thessalien, Mitteillungen, Bd. 52(1927), S. 125.

5. Damals wurde noch unter Justinian I. die beiden Städte befestigt; E. Kirsten, I, 1, S. 293.

6. E. Kirst e n, II, 1, S. 256.

7. Ebda, S. 280 ff; die Privilegien: F. M i k 1 o s i c h - J. M ü 11 er, Acta et diplomata graeca medii aevi sacra et profana, V, S. 260 ff.; E. Fr a n c è s, La féodalité et les villes byzantines au XIIIe et au XIVe siècle. «Byzantinoslavica»,Bd. 16(1955), S. 93; A. V. Solov v ev, Fessalskije archonty v XIV veke. Čerty feodalizma v vizantijskoserbskom stroje, «Byzantinoslavica», Bd. 4 (1932), S. $163 \mathrm{ff}$. 
und Joannina gilt, dann liesse sich in Phanarion auch eine handwerkliche Produktion für den lokalen Markt vermuten ${ }^{1}$. Dies wird besonders durch die Befreiung der örtlichen Adeligen von Zollgebühren unterstützt. Wenig bekannt sind die Anfänge des im 15. Jahrhundert schon als wichtigste städtische Siedlung dieser Region bekannten Karditsa. Dieses wird im 14. Jahrhundert nur als Festung-vielleicht mit einem Suburbium-erwahnt, dies in Zusammenhang mit der Eroberung durch die Katalaner ${ }^{2}$. Eine besondere Aufmerksamkeit verdienen im südlichen Teile Westthessaliens die Klöster, welche dicht unter den östlichen Hängen von Pindos gegründet wurden. In einem Gebirgstal westlich von Phanarion kennen wir in der spätbyzantinischen Periode gleich drei wichtige Klosteranlagen: Dúsikú, Kúra und Porta. Dúsikú war stark bafestigt und im 14. Jahrhundert gegründet worden ${ }^{3}$. Auch das Klöster Porta, wenn wir nach dem Alter seiner Kirche urteilen dürfen, muss im 14. Jahrhundert gegründet oder erneuert worden $\operatorname{sein}^{4}$. In den Gebirgstälern südwestlich von Karditsa liegen zwei weitere byzantinische Klöster - Korona und Petra, deren Alter jedoch nicht genau feststellbar ist ${ }^{5}$.

In dem dünner besiedelten hügeligen Süden Thessaliens finden wir nur zwei wichtigere Siedlungen mit nichtlandwirtschaftlichen Funktionen. Dicht an den östlichen Ausläufern war es Thaumakoi, eine Festung mit antiker Siedlungskontinuität, wo aus dem 10. Jahrhundert auch ein Bischofssitz erwähnt wird ${ }^{6}$. Im 14. Jahrhundert und vielleicht schon früher bekam die Festung auch eine Bedeutung für den Handel; sie wird in jener Zeit in den Vertragen mit den Venezianern erwähnt ${ }^{7}$. In den älteren Handelsverträgen wird aus dieser Gegend ein anderer Ort genannt - das südlich von Thaumakoi liegende Ezeros (im Jahre 1116), dessen städtisches Weiterbestehen in der spätbyzantinischen Periode unbekannt ist ${ }^{8}$. Die wichtigste städtische Sied-

1. E. K i r s t e n, Die byzantinische Stadt. Berichte zum XI. Intern. Byzantinisten-Kongress München 1958, S. 38.

2. K. S e t to n, The Catalan Domination of Athens 1311-1388, Cambridge Mass. 1948, S. 58.

3. L. H e u z e y, Excursion dans la Thessalie Turque 1858, Paris 1927, Г. K ú $\rho$ к о ,

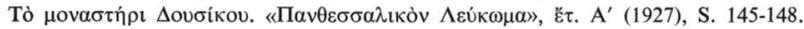

4. A. Philip pson, II, 1, S. 169.

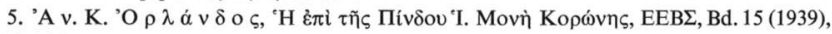
S. 405-416.

6. H. G e $1 \mathrm{z}$ er, Notitiae episcopatuum, S. 557.

7. E. Kirst e n, I, 1, S. 295.

8. L. H e u z e y, Le Mont Olympe et l'Acarnanie. Exploration de ces deux régions, avec l'étude de leurs antiquités, de leurs populations anciennes et modernes, de leur géogra-


$\lambda i \pi \varepsilon v \kappa \eta$. 
lung in Südthessalien war Pharsalos mit einer Kontinuität seit der frühbyzantinischen Zeit bis zur türkischen Eroberung. Aus dem 10. Jahrhundert kennen wir hier ein Bischoftum, aus dem 13. Jahrhundert sogar ein Erzbischoftum $^{1}$. Als eine Festung und Sitz der zivilen Verwaltung wurde Pharsalos von den Katalanern im 14. Jahrhundert besetzt; gegen Ende des Jahrhunderts wird hier nur noch eine Festung erwähnt ${ }^{2}$. Eine Handelsfunktion darf hier nur aufgrund der Siedlungsstabilität und jener Tatsache vorausgesetzt werden, dass es sich um das einzige stabile Zentrum der südthessalischen Ebene handelte. Zur handwerklichen Produktion haben wir keine Belege. Die nördlicher iegende Festung Driskoli kommt in der spätbyzantinischen Zeit schon nicht mehr vor ${ }^{3}$. Dies ist unter den alten Festungen Thessaliens keine Ausnahme.

Das hügelige Gebiet am mittleren Peneios zwischen derEbene von Trikkala und Larissa hatte in der hier untersuchten Periode kein wichtigeres Zentrum und kannte auch in den früheren Zeiten von den nichtlandwirtsschaftlichen Funktionen vielleicht nur die militärische. Mit der zeitweiligen Bedeutung der mittelthessalischen Schwelle als Grenze hängt die Häufung byzantinischer Festungen in dieser Gegend zusammen. Es waren Grisanon im Norden, Limnaion und Vlochos in den Peneios-Sümpfen, Pharkadon und Atrax, die an antike Städtesiedlungen anknüpften, und Petrinon im Süden ${ }^{4}$. Von allen diesen Festungen, von denen wir nicht sagen können, ob sie tatsächlich alle parallel oder zum Teil nacheinander funktioniert haben, zeigt ein städtisches Ausmass und Siedlungscharakter nur Petrinon - dies jedoch aus der frühbyzantinischen Zeit ${ }^{5}$. Aus der späteren Periode kennen wir keine eindeutigen Zeugnisse über das Bestehen dieser Festungen; sie werden auch nicht bei jenen Autoren genannt, welche, wie Kantakuzenos, ein besonderes Auge für die militärischen Anlagen hatten. Wahrscheinlich haben sie durch die politischen verschiebungen ihre Bedeutung verloren. Bei einigen von ihnen blieb eine kontinuierliche Dorfsiedlung bis in die Neuzeit (Grisanon, Pharkadon).

Das wichtigste Zentrum Thessaliens war während der ganzen byzantinischen Zeit nhne Zweifel Larissa. IIier kunzentrlerten sich alle wesentlichen nichtlandwirtschaftlichen Funktionen, die wir verfolgen (natürlich mit Ausnahme des Seehandels). Hier war die kirchliche Metropolis für ganz Thessalien und auch ein erstrangiger Sitz der zivilen Verwaltung ${ }^{6}$. Hier trafen sich

1. H. G elz er, Notitiae episcopatuum, S. 592; I. 'A $v \delta \rho \varepsilon$ ó $\pi$ o v $\lambda$ o 与, 'Е $\pi \alpha \rho \chi i ́ \alpha$

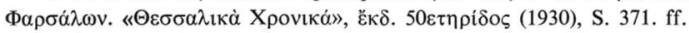

2. K. M. S e t t o n, Catalan Domination, S. 29; E. K i r s t e n, I, 1, S. 296.

3. Ebenda, S. 278.

4. A. Phili p p s o n, I, 1, S. 54, 61, 67; E. K irsten, Ebenda, S. 260 u. 293.

5. Ebenda, S. 278 u. 293.

6. H. G e lz er, Notitiae episcopatuum, S. 551, 598; $\Delta . \mathrm{X} \alpha \tau \zeta \eta \gamma \iota \dot{\alpha} \vee v \eta \varsigma$, 'A $\pi$ ò 
auch die Fernhandelswege aus Epirus über Trikkala, aus Thessaloniki und aus dem Süden. Bei Idrisi wird Larissa als eine glorreiche und mächtige Stadt charakterisiert, in deren Umgebung Wein, Getreide und Feigen im grossen Ausmass produziert werden ${ }^{1}$. Für die ökonomische Funktion der Stadt ist es bezeichnend, dass sich in ihren Mauern dauernd eine jüdische Gemeinde aufhielt ${ }^{2}$; daneben werden hier im 14. Jahrhundert auch Vlachoi, Bulgaren und Albaneser erwähnt ${ }^{3}$. Die dramatischen politischen Wandlungen, welche die Stadt während der spätbyzantinischen Zeit erleben musste, sind ein Zeugnis ihrer ausserordentlichen Bedeutung auch in jener Periode; das handelspolitische Interesse der Venezianer hat sich deutlich im 14. Jahrhundert gemeldet $^{4}$. Die heute erforschte reiche kirchliche Architektur gehört auch zu den indirekten Beweisen einer wirtschaftlichen Blüte von Larissa im Mittelalter, während man aus der türkischen Zeit eher von einem steigendem $\mathrm{Fe}$ stungscharakter erfährt ${ }^{5}$. Larissa hat augenscheinlich die ausseragarrischen Funktionen für ihre weite Umgebung übernommen. Die in ihrer Nähe situierte antike und frühbyzantinische Festungsstadt Mylai verschwand sehr bald aus den Quellen ${ }^{6}$ und die Nachbarstädte wie Pharsalos und Golos sind ziemlich weit entfernt; das am nächsten liegende Tyrnavos stammt erst aus der späteren Zeit.

Der hügelige Norden Thessaliens war ziemlich dünn besiedelt und hatte sein altes Verwaltungszentrum in Elasson, einem Bischofssitz mit alter Siedlungstradition schon aus der spätrömischen Zeit; shon Kaiser Justinian liess die Stadtmauern erneuern, bzw. einrichten. Später hat hier der Kaiser Andronikos II ein Erzbischoftum gegründet? ${ }^{7}$ Im 14. Jahrhundert wird sonst die

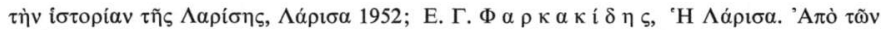

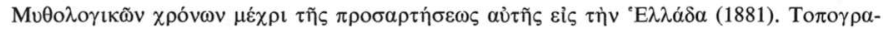

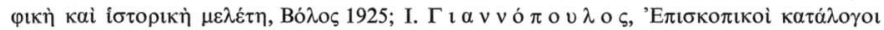



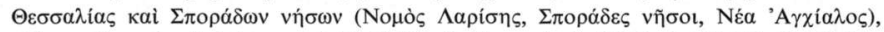

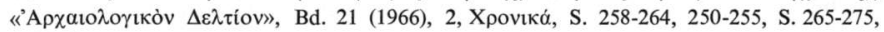
256-272.

1. W. To m a s ch e k, Idrisi, S. 348 ff. u. 350 .

2. N. Bees, Übersicht über die Geschichte des Judentums von Joannina, «ByzantinischNeugriechische Jahrbücher», Bd. 11 (1921), S. 159 ff. N. I. $\Gamma ı \alpha v v$ ó $\pi$ o $v \lambda \circ \varsigma, \Sigma v \mu \beta \circ \lambda \alpha i$

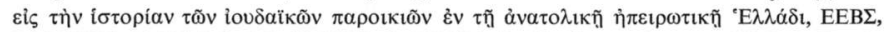

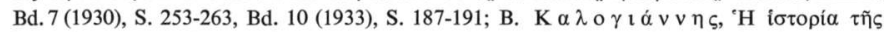

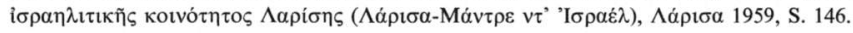

3. F. D öl g e r, Regesten III, S. 23.

4. E. Kirste n, I, 1, S. 295 ff.

5. Neue archäologische Ausgrabungen, П. $\Lambda \alpha \zeta \alpha \rho i \delta \eta$, a.a.O. Anm. 31.

6. A. Philippson, I, 1, S. 85.




Bedeutung als eine Festung betont ${ }^{1}$. Die Funktion einer Handelsstation darf von der wichtigen Lage auf dem Handelswege nach Makedonien abgeleitet werden; fraglich bleibt das Ausmass des lokalen Marktes. Für einen lokalen Markt scheint jedoch die Tatsache zu sprechen, dass von den antiken Städten aus dieser Gegend, wie Azoros, Maloia, Leimoné und Domenikos (antikes Chyreitai) ${ }^{2}$, nur Elasson in das Mittelalter überlebte, ein Ort, der als einziger von den genannten eine fruchtbare Umgebung hatte. Neu hat sich erst in der spätbyzantinischen Periode im Süden in einer landwirstschaftlich reichen Ebene Tyrnavos entwickelt, dessen Blüte dann besonders in die türkische Zeit gehört ${ }^{3}$.

Der Nordosten Thessaliens ist geographisch durch grosse Kontraste charakterisiert; einerseits das reiche Peneios-Tal und die Ebene südlich von OssaGebirge, andererseits dieses Gebirge und der Nieder-Olymp. Im weiten Norden kennen wir aus der spätbyzantinischen Zeit die Festung Petra, unter der eine kleine Siedlung und in deren Nähe ein Kloster lag. Eine bedeutendere Handelsfunktion als jene der Handelsstation ist hier kaum vorauszusetzen ${ }^{4}$. Die am Ufer liegende Festung und ehemals antike Stadt Dion ist in der uns interessierenden Zeit schon unbekannt ${ }^{5}$. Die wichtigste städtische Siedlung dieser Zeit ist unter den Olympos-Hängen Platamonas geworden; eine Burg und Siedlung am Meeresufer. Die ersten Anfänge ihrer Blüte stammen schon aus dem 12. Jahrhundert, wo sie eine wichtige strategische Position (an der schmalsten Stelle zwischen Olympos und dem Seeufer auf dem Wege nach Makedonien) mit einer Handelsfunktion verbunden hat ${ }^{6}$. Schon am Ende des 12. Jahrhunderts haben hier die Venezianer wichtige Positionen gewonnen? Ein bedeutender Markt ist hier jedoch kaum vorstellbar wegen der geographischen Lage. Im 14. Jahrhundert wird Platamonas bei Kantakuzenos als

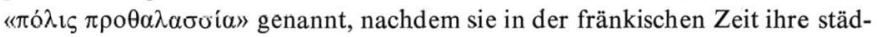

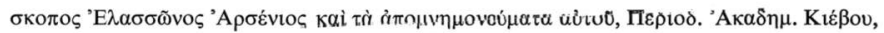

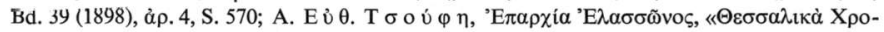



1. Cant., Bonn; I, S. 474; L. H e u z e y, Le Mont Olympe et l'Acarnanie, S. 25-26.

2. A. Philip pso n, I, 1, S. 80 ff., E. Kirst e n, Ebenda, S. 293.

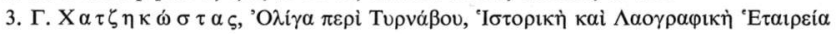

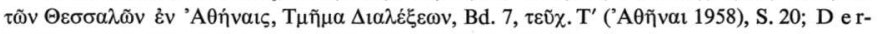

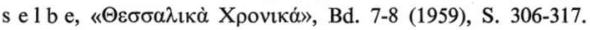

4. L. H e u z e y, Le Mont Olympe, S. 148.

5. A. Philip pson, I, 1, S. 105; E. Kirst e n, Ebda, S. 293.



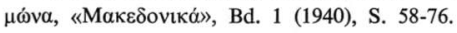

7. G. T a f e l-G. Tho m a s, Urkunden zur älteren Handels - und Staatsgeschichte der Republik Venedig I. Theil (814-1205), Wien 1856, S. 267., 
tischen Funktionen erfolgreich bewahrt hatte ${ }^{1}$. Auch in der türkischen Zeit blieb ihr eine bedeutende strategische und handelspolitische Funktion ${ }^{2}$. Sie war also mehr als eine einfache Handelsstation und konnte als Hafen und eventuell auch Handelsplatz für Larissa gedient haben; eine Funktion, welche in der frühbyzantinischen Zeit für Larissa der südlicher liegende Hafen (und Festung) Stomion ausgeübt hatte. An der Stelle von Stomion, das wir in den späteren Quellen nicht finden, erscheint in den italienischen Seekarten ein Hafen Monastir Aidimitri, dessen Name wahrscheinlich im Zusammenhang mit dem über dem alten Stomion gegründeten Kloster Hagios Demetrios steht ${ }^{3}$. Auch die weiter südlich liegende Karytsa, das Idrisi noch als Städtchen erwähnt, ist aus der späteren Zeit unbekannt ${ }^{4}$.

Im Peneiostal zwischen dem Nieder-Olymp und Ossa kennt man einige Festungen, von denen die mit Larissa verbundene Burg Halmyros aus der Palaiologenherrschaft schon nicht mehr bekannt ist ${ }^{5}$, während Lykostomion in der spätbyzantinischen Zeit neue Funktionen übernahm. Es war nicht nur eine Festung, sondern auch Bischofssitz und ein Handelszentrum, in dessen Nähe Salzgruben arbeiteten ${ }^{6}$. Eine Sonderstellung im nordöstlichen Thessalien hatte das fruchtbare Tal Hagia zwischen den Gebirgsmassiven von Ossa und Mavrovuni. Dieses Gebiet hatte ein archäologisch festgestellstes Marktzentrum aus der byzantinischen Zeit, dessen Name nicht bekannt ist. Es ist nicht ausgeschlossen, dass es mit der aus der frühbyzantinischen Periode bekannten städtischen Siedlung und Festung Kentavropolis identisch ist ${ }^{7}$. Die einzige Ortschaft, welche sich die wesentliche ihrer Funktionen bis in das 14. Jahrhundert bewahrt hat, war in dieser Region Kastri (antikes Kerkineion), einer der Stützpunkte der byzantinischen Herrschaft im 14. Jahrhundert nach der zeitweiligen Eroberung durch die Katalaner ${ }^{8}$. Wahrscheinlich

1. L. H e u z e y, Le Mont Olympe, S. 89-90; C a n t., Bonn, II, S. 355.

2. L. H e u z e y, L'expédition, S. 90.

3. A. Philip pson, I, 1, S. $114 \mathrm{ff}$.

4. E. K irs t e n, Beiträge, I, 1, S. 295.

5. Th. L. F r. T a f e 1, Symbolarum criticarum geographiam Byzantinam spectantium partes duae. Pars prior, München 1849, Bayer. Akad. der Wissensch. Abhandl. der hist. Cl., Bd. 5, Abt. 2, S. 68, 473, 495; J. L o n g n o n, Les noms de lieux de la Grèce franque, Journal Savants Juill. 1960, S. 100; E. K ir s t e n, Beiträge, I, 1, S. 294.

6. Ca nt., Bonn, I, S. 474; D ukas, Bonn, S. 341; A. S o lov j e v-V. M oš i n, Grčke povelje srpskich vladara, Fontes rerum slavorum meridionalium. Diplomata graeca regum et imperatorum Serviae. I. Zbornik za istoriju jezika i kniževnost srpskog naroda, kn VII, Beograd 1936, S. 226, XX, S. 158.


Bónos 1948, S. 32.

8. K. S e t t o n, Catalan Domination, S. $106 \mathrm{ff}$. 
ist es identisch mit dem «Städtchen» Kastri in Thessalien, welches bei Kantakuzenos erwähnt wird ${ }^{1}$. Von den wirtschaftlichen Funktionen dieser Siedlung ist aus dieser Zeit nichts bekannt.

Den südostlichen Teil Thessaliens können wir in drei Regionen einteilen: die Halbinsel Magnesia mit der Golos (Volos) - Ebene, die Halmyros - Ebene und das Gebirge Othrys. Magnesia mit dem Pelion-Gebirge war erst in der spätbyzantinischen Periode dichter besiedelt, besonders auf den westlichen Hängen, wo im 13. und 14. Jahrhundert einige Klöster mit den in ihrer Nähe neu gegründeten Dörfern entstanden sind. Von anderen ausseragrarischen Funktionen wissen wir jedoch im gebirgigen Binnenland fast nichts. Im Süden der Halbinsel überlebten aus der Antike am Ufer zwei kleine städtische Siedlungen in die byzantinische Zeit; der Hafen Aphétai und das Städtchen Olizon. Im Gebirge kennt man eine byzantinische Burgruine ${ }^{2}$. Aus der uns interessierenden Zeit kennen wir diese Ortschaften schon nicht mehr. Die städtischen Funktionen haben sich in der späteren Zeit im Norden konzentriert - in der kleinen aber reichen Ebene am Ufer zwischen den Ziragiotischen Höhen und dem Pelion-Gebirge. In der frïhbyzantinischen Periode kennen wir hier einen Handelsplatz und Hafen mit der Siedlungskontinuität seit der hellenistischen Epoche, Demetrias. Im 10. Jahrhundert ist hier ein Bischofssitz erwähnt ${ }^{3}$. Bei Idrisi wird Demetrias als eine menschenreiche Hafenstadt und der wichtigste Exporthafen für thessalisches Getreide charakterisiert ${ }^{4}$. Aus der spätbyzantinischen Zeit kennen wir aber diesen Ort vor allem als eine Festung, für welche sich die Latiner und Katalaner lebhaft interessierten und in deren Umgebung sich viele Mühlen befanden ${ }^{5}$. Das unweit liegende Kloster Theometor Makrinitissa hat sich im 13. Jahrhundert am Schiffsbau beteiligt ${ }^{6}$. Gegenüber dem Hafen von Demetrias war auf der anderen Seite der Bucht in der frühen byzantinischen Zeit ein anderer Hafen tätig, Palatia, der noch im 13. Jahrhundert regelmässige Handelsbeziehungen zum $\mathrm{Hl}$. Johannes-Kloster auf der Insel Patmos unterhielt. Der Hafen verschwan jedoch imfolgenden Jahrhundert und cs blieb an seiner Stelle nur ein Dorf mit slavischen Einwohnern? ${ }^{7}$. Der Rückgang an Bedeutung der beiden Hafen-Demetrias und Palatia - hängt ohne Zweifel mit dem Aufstieg eines dritten zusammen: während

1. C a n t., Bonn, III, S. 130; L. H e u z e y, Le Mont Olympe, S. 87.

2. A. Philippson, I, 1, S. $141 \mathrm{ff}$., $150,160 \mathrm{f}$.

3. H. G e 1 z er, Notitiae episcopatuum, S. 557.

4. W. To m a s c h e k, Idrisi, S. 351.

5. W. M i 11 e r, The Latins in the Levant, London 1908, S. 305; K. S e t t o n, Catalan Domination, S. 105, 315; T a f e l- T h o m a s, Urkunden, FRA III, S. 195.

6. F. D ö $1 \mathrm{~g}$ e r, Regesten III, S. 58; MM IV, S. 426.

7. F. D öl g e r, Regesten III, S. 4. Die Existenz dieser Lokalität ist problematisch. 
der 1. Hälfte des 14. Jahrhunderts hören wir ofter den Namen Golos als Bezeichnung eines Hafens mit Festung und Fernhandelsbeziehungen ${ }^{1}$. Seine Bedeutung stieg ganz eindeutig und hat sich auch während der Türkenzeit vergrössert - damals schon unter dem Namen Volos ${ }^{2}$.

Eine ähnliche Verschiebung der städtischen Funktionen finden wir in der Phtiotis. Seit der frühen Zeit bis in das 7. Jahrhundert konzentrierte sich die politische Verwaltung und der Handel in der Stadt Thebai Phthiotides ${ }^{3}$. Die fränkischen Eroberer haben im 13. Jahrhundert die Stadt neu befestigt und die militärische Funktion hat sich dann noch in dem Kampf mit den Katalanern bewährt ${ }^{4}$. Nach der türkischen Eroberung war der Verfall vollendet. Das Schicksal von Thebai teilte auch sein Hafen Pyrassos, der schon früher als es seine Handelsfunktion einbüsste und nicht mehr in den Quellen vorkommt ${ }^{5}$. Während des 12. Jahrhunderts (und vielleicht schon früher) begann sich die Vermittlung des Handels und des Transports an eine bisher unbekannte Lokalität zu verschieben. Sie hiess ursprünglich Halos und bekam jetzt den Namen Halmyros. Auch wenn es bisher nicht klar ist, ob es sich um eine Namens oder auch eine Siedlungsverschiebung handelte, besteht kein Zweifel daran, dass Halmyros gegen Ende des 12. Jahrhunderts schon zu einem wichtigen Hafen wurde. Idrisi bezeichnete ihn als ein wichtiges Emporium, besucht von fränkischen und byzantinischen Kaufleuten ${ }^{6}$. Ähnliches berichtete auch Benjamin von Tudela, der hier darüber hinaus noch eine jüdische Gemeinde von unglaublich hoher Anzahl, nämlich 400 Mitgliedern kannte? Aus dem Jahre 1199 stammt die erste Erwähnung in dem Handelsvertrag zwischen Byzanz und den italienischen Kaufleuten über Getreideausfuhr aus den byzantinischen Hafen ${ }^{8}$. Im Jahre 1265 haben die Venezianer eine Handelsniederlassung in Halmyros, von dem Kaiser bekommen und haben auch später ihre

1. E. K ir s te n, Beiträge I, S. 296; Can t., Bonn, I, S. 434. N. I. $\Gamma \iota \alpha v v$ o $\pi$ o v́ $\lambda$ o v,

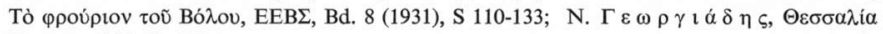

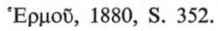



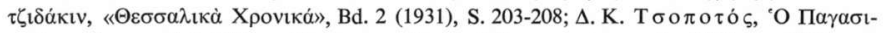

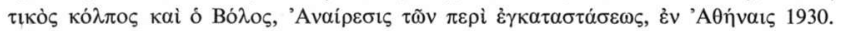





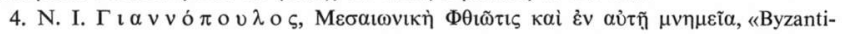
nische Zeitschrift» (1927), S. 27 u. 46 f.

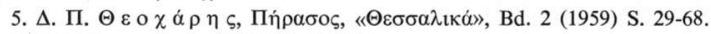

6. W. T o m a s ch e k, Idrisi, S. 348 .

7. J. S t a r r, The Jews in the Byzantine Empire 641-1214, Athens 1939, S. 230.

8. E. Kirst e n, Beiträge, I, 1, S. 279. 
wichtige Position behalten ${ }^{1}$. Trotz der katalanischen Plünderung lebte der Hafen auch im 14. Jahrhundert weiter, jedoch aber es scheint ${ }^{2}$, dass sich ein Teil seines Handels in den damals aufkommenden Golos allmählich verschoben hat ${ }^{3}$. Während der türkischen Besatzung hat sich die Siedlung in das Binnenland zurückgezogen; der ehemalige Ort Kirtsini bekam den Namen Halmyros ${ }^{4}$. In der Lokalisierung und Namensverschiebung ist bisher manches unklar geblieben ${ }^{5}$. Auf jeden Fall dürfen wir aufgrund der sich zwar verchiebenden, sich aber doch zähe haltenden Fernhandelsfunktion in dieser Gegend mit gewisser Sicherheit voraussetzen, dass die Zentren des Fernhandels zugleich die Funktion der Lokalmarkte für das agrarische Hinterland ausgeübt haben. Dabei konnten die verfallenden Zentren des Fernhandels wie Thebai, Demetrias oder zuletzt Halmyros ihre Funktion des lokalmarktes durchaus weiter behalten.

In dem Orthrys-Gebiet finden wir Elemente der nichtlandwirtschaftlichen Tätigkeit nur am östlichen und südlichen Uferstreifen. Am Eingang in den Golf von Volos entstand schon in der Antike ein Hafen, der auch in der byzantinischen Epoche seine Bedeutung behalten hat: Pteleon. Bedeutend war eher seine Lage als sein wirtschaftliclies Hinterland. Im 13. Jahrhundert und dann wieder am Anfang des 14. Jahrhunderts haben ihn die Venezianer erobert, um ihn bis 1470 als einen strategischen Punkt zu halten, gegen die Serben, Katalaner, Albanesen und zuletzt gegen die Türken ${ }^{6}$. Um die Mitte des 15. Jahrhunderts sprach man jedoch allgemein von dem schlechten Hafen wegen seiner schlechten Lage und wegen des schwierigen Zugangs in den Hafen ${ }^{7}$. Südwestlich von Pteleon lag unter dem Othrys-Gebirge eine kleine Stadt, die in der ehemaligen Akropolis der untergangenen antiken Stadt Larissa Kremaste gegründet wurde: Gerdikion. Im 12. Jahrhundert hat es Benjamin von Tudela als eine verfallende Stadt mit geringer Einwohnerzahl und

1. F. D ölg er, III, 8, 48.

2. L. B r e h i e r, Vie et mort de Byzance. Le Monde Byzantin I, Paris 1947, S. 421.

3. Über die Beziehungen der beiden Orte vgl. N. G i a n no poulos, D’Halmyros à Volos, «Revue des Études anciennes», Bd. 35 (1933), S. $329 \mathrm{f}$.

4. E. Kirst e n, Beiträge, I, 1, S. 296.





6. K. S e t t o n, The Catalan Domination, S. 30 u. 254; G. Th o m as - R. Pr ed e 11 i, ed. Diplomatarium Veneto-Levantinum (DVL), Venise 1880, I, S. 218; N. J or g a, Notes et extraits pour servir à l'histoire des croisades au XVe siècle: Documents politiques, «Revue Orient latin», Bd. 4 (1896), S. 511.

7. F. T h ir i e t, Régestes des délibérations du Sénat de Venise concemant la Romanie (1329-1463), Paris 1958-1961, III, S. 171. 
einer kleinen jüdischen Gemeinde beschrieben ${ }^{1}$. Am Anfang des 14. Jahrhunderts wurde sie von den Katalanern überfallen; sie war also trotz des Rückangs ihrer Bedeutung noch interessant für die Eroberer ${ }^{2}$. Die Funktion des Lokalmarktes ist damals wohl schon ohne Bedeutung gewesen, auch von dem Anschluss an die Fernhandelswege kann kaum die Rede sein. Seit der frühen Periode bis in das 13. Jahrhundert hinein berichten die Quellen über die Existenz der Festung Egna (des antiken Achinos) nahe des südlichen Meeresufers des Othrys-Gebietes; es residierte dort auch der Bischof. Ihre Aufgabe war vor allem, den Zugang zur Spercheios-Mündung zu schützen³. Seit dem 13. Jahrhundert werden doch nur die kirchlichen Funktionen von Egna erwähnt ${ }^{4}$.

Wir haben hier diejenigen thessalischen Orte untersucht, die wir als Träger der ausseragrarischen, städtischen Funktionen betrachten. Wir konnten dabei eine sehr unterschiedliche Intensität solcher Funktionen und eine Variabilität ihrer Kombinationen feststellen. Es gab einerseits spät im byzantinischen Thessalien Orte, für die nur eine der nichtlandwirtschaftlichen Funktionen zutraf, die sich also nur in einer Beziehung von den einfachen Dörfern unterschieden haben. Zu dieser Gruppe gehöı en die Festungen und die Klöster; die ersteren haben während der spätbyzantinischen Zeit (oder schon früher) stark abgenommen, die anderen zugenommen. Andere als militärische oder kirchliche Funktionen treten nur ausnahmsweise auf, wie z. B. beim Hafen Monastir Aidimiri oder beim Markt Raxa. Auf der anderen Seite konnten wir in mehreren Fällen eine Anhäufung von fast allen wesentlichen ausseragrarischen Funktionen in einem spätbyzantinischen Ort feststellen. Hierher, gehören die wichtigsten Städte Thessaliens, wie Larissa, Halmyros, Trikkala, Phanarion und mit Hinblick auf die weitere Entwicklung auch Golos-Volos. Wenn diesen Städten die direkten Kontakte mit dem Fernhandel fehlen (vielleicht Ausnahmevon Halmyros), dann entspricht das dem allgemeinen Charakter der ökonomischen Entwicklung Thessaliens zum Unterschied z. B. von Epirus und von einigen Küstenstreifen der Peloponnes.

Zwischen den beiden Typen - dem «monofunktionellen» und dem einer «vollwertigen» Stadt - finden wir die stadtähnlichen Siedlungen, in denen sich zwar mehrere, nicht aber alle der wesentlichen städtischen Funktionen kombinierten. Meistens handelte es sich um eine Koppelung der militärischen und ökonomischen Funktion; einige Festungen waren zugleich wichtige Zen-

1. J. S t a r r, The Jews, S. 230 .

2. K. S e t t o n, The Catalan Domantion, S. 29.

3. H. G e l z er, Notitiae episcopatuum, S. 557.

4. A. Phili p p s o n, I, 1, S. 205. 
tren des Fernhandels (Platamonas, Thaumakoi, Golos in seinen Anfängen), andere, eher die kleineren, hatten gleich Lokalmarkt (Karditsa, Gardikion, vielleicht auch Kastri), in einem Falle hatte die Festung zugleich einen Durchgangshafen (Pteleon). Mehrfach verband sich die militärische Funktion mit jener der zivilen oder kirchlichen Verwaltung; dort, wo die Ortschaft zugleich auf einem Handelswege liegt, dürfen wir noch eine Handelsfunktion voraussetzen (Lykostomion, Elasson, Stagoi, Pharsalos). Mit dem oben erwähnnen Vorbehalt dürfen wir alle zu dieser dritten Gruppegehörenden Siedlungen als Städte, Kleinstädte oder stadtähnliche Siedlungen bezeichnen. Wenn wir alle als Stadt bezeichnete Lokalitäten in die Karte projizieren, dann stellen wir fest, dass wir in der spätbyzantinischen Zeit in allen fruchtbaren Gebieten bis auf eine Ausnahme (das Tal Hagia) wenigstens eine Stadt finden. Hier wird ein weiteres Zeugnis dafür geliefert, dass sich die landwirtschaftliche Marktproduktion auch ausseragrarische Funktionen schafft, bzw. nach ihnen fragt. Diese Feststellung werden wir plastischer illustrireren und belegen können, wenn wir den dynamischen Aspekt in Betracht ziehen und die Änderungen in der städtischen Besiedlung untersuchen.

Wenn wir aufgrund der oben zesammengestellten Angaben die städtische Besiedlung Thessaliens in der früheren und in der palaiologischen Zeit vergleichen, stellen wir wesentlich Unterschiede fest. Besonders auffallend, wenn auch nicht für das Städtewesen so bedeutend, ist die Reduzierung der Festungsanlagen; sie verschwinden entweder völlig odeı verlieren ihren Festungscharakter und verwandeln sich in einfache Dorfsiedlungen. Von den alten Festungen blieben in die spätbyzantinische Zeit in ihrer Bedeutung als Festung meistens diejenigen, welche zugleich noch eine andere Funktion ausübten, wie Platamonas, Lykostomion, Elasson, Pharsalos usw. Nur selten wurden neue Festungen angelegt und diese kennen wir eventuell bald auch als städtische Zentren, wie es in dem Falle von Karditsa gewesen ist. Für die städtische Besiedlung von grosser Bedeutung war die Tatsache, dass eine zahlreiche Reihe von frühbyzantinischen Städten nicht in der von uns untersuchten Zeit auffindbar oder in ihrer Funktion belegbar war. Andererseits sind mehrere neue städtische Siedlungen entstanden oder haben neue Funktionen übernommen. Auch wenn rein quantitativ gesehen die Anzahl der zurück - bzw. untergegangenen städtischen Siedlungen grösser ist als die der neuentstandenen, wäre es falsch daraus auf einen Rückgang der städtischen Besiedlung oder des städtischen und wirtschaftlichen Lebens Thessaliens in der spätbyzantinischen Zeit zu schliessen. Wir haben nämlich kein greifbares Kriterium füI die Intensität der in den einzelnen untergegangenen oder neu entstandenen Siedlungen vertretenen ausseragrarischen Funktionen.

Wenn wir die Entwicklung in den einzelen Regionen genauer untersu- 
chen, dann stellen wir fest, dass überall, wo eine alte Stadtsiedlung in der spätbyzantinischen Periode nicht mehr vorkommt, eine neue Stadt in ihrer nähe entstanden ist, oder eine ältere Ortschaft neue städtische Funktionen ausübt. Wir dürfen wolf hypothetisch festsellen, dass es zu Verschiebungen der städtischen Funktionen kam; während die Stadtbesiedlung in Thessalien im Laufe der Jahrhunderte eher unstabil als stabil war, haben sich die städtischen Funktionen überraschend stabil erwiesen. So hat z. B. Elasson die Funktionen von Maloia und Leimone übernommen, die Funtktion von Stomion ist in Platamonas zu finden, die von Metropolis und Gomphoi in Phanarion und eventuell auch in Karditsa. In besonders «reinen» Formen gingen diese Funktionsverschiebungen an dem Golf von Volos vor sich; von Thebai Phthiotides auf Halmyros, don Demetrias auf Golos und später noch von Halmyros zum Teil auf Golos. Wir haben in diesem Aufsatz die komplizierte Problematik der ökonomischen Funktionen der Klöster beiseite gelassen; es darf jedoch nicht übersehen werden, dass die neuen Klöster in Gebieten mit grösserer Nachfrage nach Ausübung der handelsvermittlenden Funktionen entstanden sind. Auch der Anteil der Klöster an der ausseragrarischen Produktion wäre noch zu untersuchen.

Thessalien ist ein allzu kleiner Teil Mittel-Südosteuropas, um uns zu breiteren Verallgemeinerungen zu berechtigen. Wir möchten uns daher mit der Feststellung begnügen, dass wir in Thessalien diejenigen allgemeinen Regelmässigkeiten in der Verschiebung der städtischen Funktionen gefunden haben, die wir auch für andere griechische Landschaften konstatierten ${ }^{1}$. Die eine Regelmässigkeit scheint darin zu bestehen, dass die Verschiebung der stäđtischen Funktion näher zum Gebirge zielt; dies war in Elasson und Tyrnavos, in Lykostomion, in Stagoi und eventuell auch bei den west-thessalischen Kösitern der Fall. Von den wenigen stabilen Städtesiedlungen, die eine durchgreifende Kontinuität aufweisen, war nur die wichtigste von ihnen - Larissa in einer breiten Ebene situiert, während sonst eben die näher zum Gebirge liegenden Orte überlebten (Pharsalos, Trikkala, Thaumakoi). Die geographische Lage war natürlich nur einer der Faktoren, sie konnte allein nicht entscheiden und es wird erst zu untersuchen sein, warum die Lage näher zum Gebirge eine positivere (wenigstens in einigen Fällen) Auswirkung hatte. Der zweite der für die Verschiebungen relevanten Zusammenhänge kommt in Thessalien seltener vor; es ist die Anziehungskraft der Fernhandelswege, die wir besonders am Meeresufer feststellen können.-Abschliessend sei

1. V. H ro chová, Byzantskà města ve 13-15. stoleti, Přispěvek k sidelni topografii středověkého Řecka, Acta Universitatis Carolinae Phil et Hist., Monographia XX, 1967, S. $89 \mathrm{f}$. 
konstatiert, dass die fehlende zweite gesellschaftliche Arbeitsteilung zwar die Analyse der städtischen Entwicklung erschwert, sie jedoch nicht unmöglich. macht. Die hier beobachtete relative Unstabilität der städtischen Siedlungen ist wohl auf die fehlende zweite Arbeitsteilung zurückzuführen. Die relative Stabilität der städtischen, ausseragrarischen Funktionen zeigt jedoch, dass ein städtisches Leben auf einem gewissen Niveau der Warenproduktion auch ohne voll durchgeführte zweite gesellschaftliche Arbeitsteilung möglich ist. Dies sind jedoch alles Betrachtungen, die erst aufgrund der vergleichenden Untersuchung der städtischen Besiedlung anderer byzantinischen Regionen nachgeprüft werden müssen. 


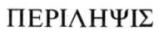

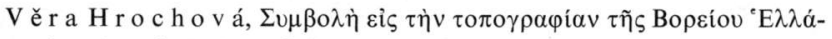

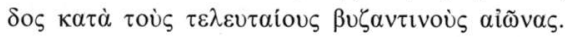



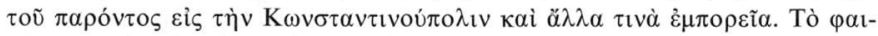



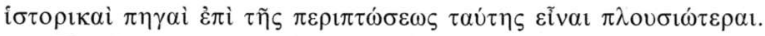

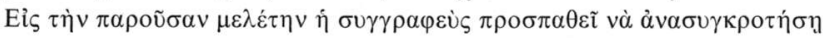

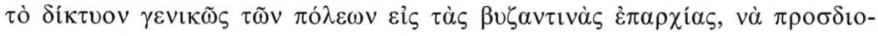

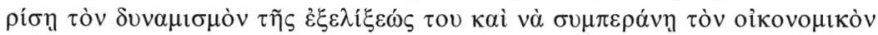

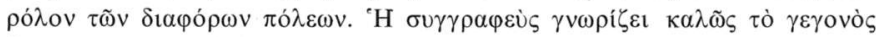

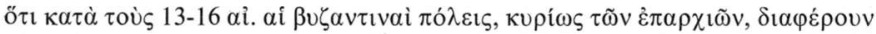

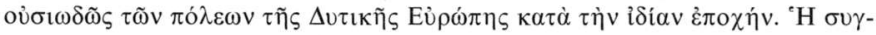

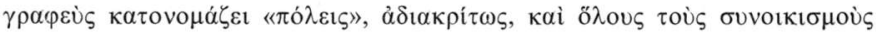



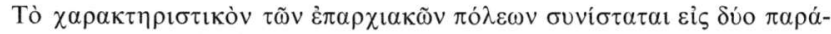







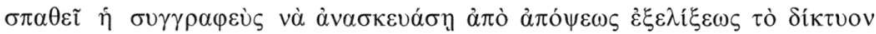

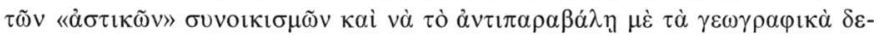



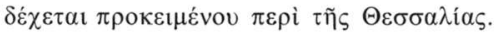

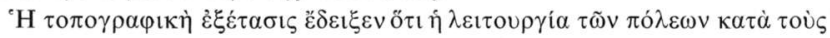

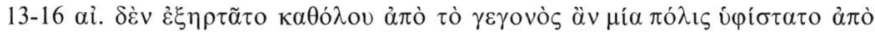

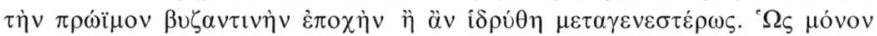



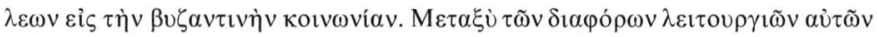



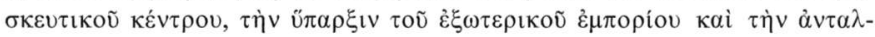



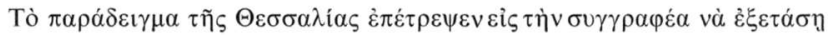

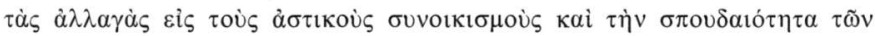

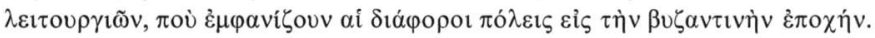

